\title{
Effects of Wavelength on Strain-Induced Birefringence of Polymers
}

\author{
Tadashi Inoue, Shozo Kuwada, Deug-Soo Ryu, ${ }^{\dagger}$ \\ and Kunihiro OSAKI \\ Institute for Chemical Research, Kyoto University, Uji, Kyoto 611-0011, Japan
}

(Received April 30, 1998)

\begin{abstract}
Strain-induced birefringence, $\Delta n$, was measured at wavelengths $400 \mathrm{~nm}<\lambda<800 \mathrm{~nm}$ for uniaxially elongated films of polystyrene (atactic and syndiotactic), bisphenol A polycarbonate, poly(ethylene terephthalate), and poly(ethylene naphthalate). The magnitude of $\Delta n$ was always higher at lower $\lambda$ : the reduced birefringence, $k(\lambda) \equiv \Delta n(\lambda) / \Delta n(633 \mathrm{~nm})$, was a decreasing function of $\lambda$. The strain-induced crystallization in poly(ethylene terephthalate) (PET) and syndiotactic polystyrene (sPS) did not significantly affect $k(\lambda)$. For polycarbonate (PC) and PET $\lambda$-dependence was much stronger when the strain was applied in the melt state (corresponding to the stress-optical coefficient, SOC) than in the glassy state (corresponding to the photoelastic coefficient, PEC); the difference was very small for atactic polystyrene (aPS). These results may be explained with a model theory in which the SOC is associated solely with the chain orientation while the PEC is affected also by the rotation of repeating units around the chain axis induced by deformation of the material glassy state.
\end{abstract}

KEY WORDS Birefringence / Stress-Optical Coefficient / Photoelastic Coefficient / Polymer Film /

Strain-(or stress-)induced birefringence, $\Delta n$, is an important property of polymeric materials; it may be used for polymer rheology. ${ }^{1,2}$ It often shows a harmful effect on the optical use of polymeric materials. The birefringence as well as the refractive index may vary with the wavelength of light, $\lambda$. The $\lambda$-dependence of refractive index is due to the $\lambda$-dependence of the average polarizability of the material. Possible $\lambda$-dependence of birefringence would reflect the $\lambda$-dependence of the anisotropic component of the polarizability tensor of structural unit of the material. For polystyrene elongated at high temperatures the magnitude of $\Delta n$ decreased with decreasing $\lambda .^{3}$ The decrease is stronger than that of refractive index, $n ; n(486 \mathrm{~nm}) / n(651 \mathrm{~nm})=1.012^{4}$ and $\Delta n(486 \mathrm{~nm}) / \Delta n(651 \mathrm{~nm})=1.073$ for polystyrene. ${ }^{3} \mathrm{Re}-$ cently the $\lambda$ dependence of birefringence is reported for some polymeric liquid crystalline systems in regard to the measuring method of birefringence with white light and a spectrometer. ${ }^{5}$ Apart from these few studies, the effect of wavelength on the birefringence has not been studied in detail.

Strain-induced birefringence in the melt or the rubbery state is different from that in the glassy state. ${ }^{6}$ For the former, the ratio of the birefringence to the stress is called the stress-optical coefficient (SOC). For the latter, the ratio is called the photoelastic coefficient (PEC). For polystyrene, for example, the SOC is negative and the PEC is positive. SOC is believed to be associated with the deformation of elastic polymer chain regarded as a Gaussian chain and hence to the orientation of the chain axis., ${ }^{1,7}$ Studies on the birefringence in oscillatory deformation over the glass-to-rubber transition zone have revealed that the PEC is associated with the orientation of chain axis as well as of the rotational orientation of repeating units around the oriented chain axis. ${ }^{8,9}$

The first purpose of the present paper is to investigate the $\lambda$ dependence of $\Delta n$ for several polymers to see what it is like for polymers other than polystyrene. The second purpose is to examine the birefringence in the melt and glassy states and compare the results in view of the orientation concept mentioned above.

\section{EXPERIMENTAL}

\section{Apparatus and Method}

Birefringence was measured for an elongated polymer film with white light and a spectrometer. The film was placed between a pair of polarizing prisms and transmitted light was analyzed with a spectrometer (PMA 11 Photonic Multi-Channel Analyzer, Hamamatsu Photonics K.K.). The light source was a halogen lamp (PHL100, Mejiro Precision). The range of wavelength was $350 \mathrm{~nm}<\lambda<800 \mathrm{~nm}$. The optical train is a standard one for measurement of birefringence with a spectrometer. ${ }^{5}$

The polarization axes of two polarizing prisms were set at angles $\pi / 4$ and $-\pi / 4$ or $\pi / 4$ and $\pi / 4$ with respect to the elongation direction. The former is referred to as the crossed polarizers $(\perp)$, and the latter as the parallel (//). The intensity of the transmitted light at each $\lambda$ is related to the birefringence as follows.

$$
\frac{I_{\perp}}{I_{\perp}+I_{\|}}=\sin ^{2}\left(\frac{\pi d \Delta n}{\lambda}\right)
$$

Here $d$ is the sample thickness in the deformed state. The quantity of the left hand side oscillates between 0 and 1 as $\lambda$ varies if the retardation $\pi d \Delta n / \lambda$ is sufficiently large. Provided the retardation is known at any $\lambda, \Delta n$ at other $\lambda$ can be evaluated. $\Delta n$ can be obtained for $\lambda$ corresponding to the maximum or minimum of $I_{\perp} /\left(I_{\perp}+I_{\| /}\right)$ where the retardation is respectively an odd or even integer multiple of $\pi / 2 . \Delta n$ for intermediate values of $I_{\perp} /\left(I_{\perp}+I_{\| /}\right)$can be obtained by solving eq 1 .

As a practical procedure for evaluation, the left hand side of eq 1 measured as a function of $\lambda$ is fitted with the right hand side by assuming the $\lambda$ dependence of $\Delta n$ following Gurnee. ${ }^{3}$

$$
\Delta n=\Delta n_{0}\left(1+\frac{a}{\lambda^{2}}+\frac{b}{\lambda^{4}}\right)
$$

\footnotetext{
${ }^{\dagger}$ On leave of absence from Kolon Industries Inc., $R \& D$ Center, 212 Gong Dan-Dong, Gumi-City, Gyung Sang Buk-Do, Korea.
} 
Table I. Stress-optical coefficients, $C_{\mathrm{R}}$, and photoelastic coefficients, $C_{\mathrm{d}}$, for test polymers

\begin{tabular}{lrcc}
\hline Material & $C_{\mathrm{R}} / \mathrm{Br}$ & $C_{\mathrm{d}} / \mathrm{Br}$ & ref \\
\hline aPS & -4700 & 9.4 & 10 \\
sPS & -9500 & 3 & 11 \\
PC & 4700 & 110 & 10 \\
PET & 3360 & 120 & 12 \\
PEN & 12980 & 270 & 10
\end{tabular}

$\mathrm{Br}=10^{-12} \mathrm{~Pa}^{-1}$.

Here $\Delta n_{0}, a$, and $b$ are fitting parameters. The absolute value of $\Delta n_{0}$ increased with stress; $a$ and $b$ were independent of the stress. The quality of fitting was excellent for all specimens but those accompanied by crystallization. The results are listed as the parameters $a$ and $b$ for various polymers. For presenting the results in figures, a reduced birefringence is used.

$$
k(\lambda)=\frac{\Delta n(\lambda)}{\Delta n(632.8 \mathrm{~nm})}
$$

For samples with strain-induced crystallization, the quantity of the left hand side of eq 1 did not vary between zero and unity but between some intermediate values, as shown later. Birefringence for such a specimen was estimated from the maxima and minima of the ratio $I_{\perp} /\left(I_{\perp}+I_{\|}\right)$.

\section{Materials}

Measurement was performed for atactic polystyrene (aPS), ${ }^{10}$ syndiotactic polystyrene (sPS), ${ }^{11}$ bisphenol A polycarbonate (PC), ${ }^{10}$ poly(ethylene terephthalate) (PET), ${ }^{12}$ and poly(ethylene naphthalate) (PEN). ${ }^{10}$ Detailed studies of birefringence have been published for these materials. The stress-optical coefficients, $C_{\mathrm{R}}$, and the photoelastic coefficients, $C_{\mathrm{d}}$, are listed in Table I.

Evidently the present method cannot be applied if the material exhibits absorption of light in the studied range of wavelength. The present polymers do not exhibit absorption in the range of $350<\lambda / \mathrm{nm}<800$. The ratio of the transmitted light intensity to the incident light intensity was independent of $\lambda$ for the sample without deformation. Also the sum $I_{\perp}+I_{/ /}$for a deformed sample was very close to $I_{0}$, the transmitted intensity $I_{\| /}$on removal of the sample in all cases.

For studies on the melt state, measurement was performed in the process of elongation or stress relaxation after the elongation at temperatures higher than the glass transition temperature, $T_{\mathrm{g}}$. The same result was attained by elongating the film at a high temperature and quenching before removing the load. Most data for the high temperatures were obtained for the films quenched under the load.

For PET and sPS, the birefringence of specimens with strain-induced crystallization was studied. The film was elongated at a high temperature and held at the same length for some time. Crystallization proceeded and birefringence slowly increased in this stress relaxation process. $^{12}$ Thus a solid film of high (and known) birefringence was attained.

Birefringence for deformation in the glassy state was investigated for PS , PC, and PET. The material had to be deformed in situ because the birefringence disappeared
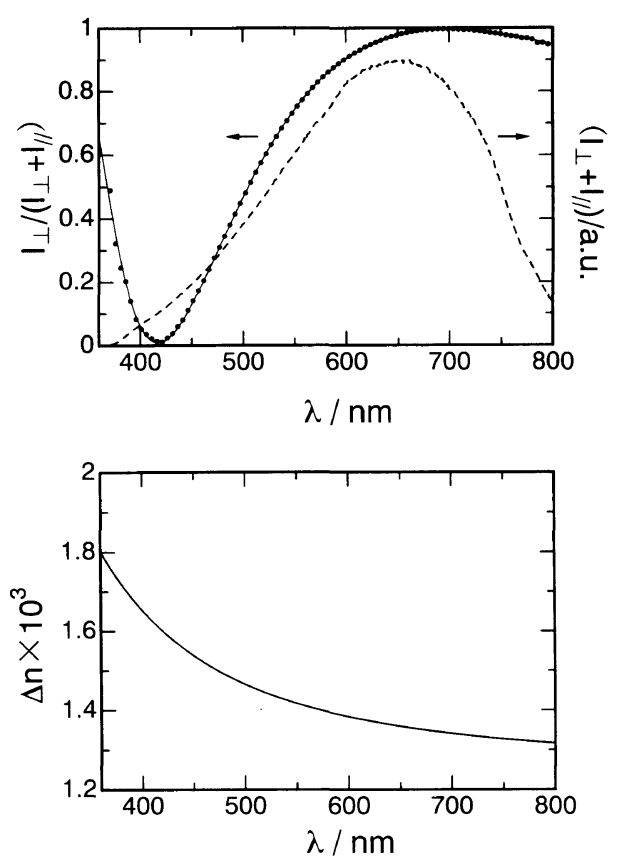

Figure 1. Results for PET film elongated at $85^{\circ} \mathrm{C}$ to an elongation ratio 1.5 and quenched. The dots represent measured results and the solid line fitting curve with eq 1 and 2 in the upper panel. $\Delta n$ of lower panel was obtained by the fitting.

on removal of the load. Since birefringence was low for PS, thick specimens, of about $3 \mathrm{~mm}$ thickness, were used and high stresses of about $5 \mathrm{MPa}$, was applied. Plastic deformations did not occur and birefringence was proportional to stress in this range of stress. Measurements were possible over wider range of conditions for PC and PET.

\section{RESULTS}

\section{Examples of Measurements}

Results for PET are shown in Figure 1 as an example of the case with relatively low birefringence. The film was elongated at $85^{\circ} \mathrm{C}$ at a constant cross-head speed corresponding to the initial elongation rate $0.025 \mathrm{~s}^{-1}$ to an elongation ratio 1.5 and then quenched. The degree of crystallization was determined as $2.6 \% .^{12}$ The sum of light intensities, $I_{\perp}+I_{/ /}$, and the ratio, $I_{\perp} /\left(I_{\perp}+I_{\| /}\right)$, are given in the upper panel. The sum $I_{\perp}+I_{\|}$agreed well with $I_{0}$, the intensity of transmitted light on removal of the specimen from the optical system. Obviously the ratio oscillates regularly between zero and unity. From the growth of birefringence during sample preparation, the maximum corresponded to $\pi d \Delta n / \lambda=\pi / 2$ and the fitting with eq 1 was an easy operation. Birefringence, $\Delta n$, is shown in the lower panel of the figure. Coefficients $a$ and $b$ are listed in Table II.

Figure 2 represents typical variation of $I_{\perp}$ at $\lambda=$ $632.8 \mathrm{~nm}$ in elongation and stress relaxation of PET leading to considerable strain-induced crystallization; elongation at $85^{\circ} \mathrm{C}$ at a constant cross-head speed corresponding to the initial elongation rate $0.025 \mathrm{~s}^{-1}$. Elongation was stopped at $80 \mathrm{~s}$ (elongation ratio $=3$; indicated with dotted line) and length was held constant thereafter. Birefringence kept increasing in elongation and stress relaxation. This may not be obvious in Figure 2 but was established through detailed evaluation of 
Table II. Fitting parameters $a$ and $b$ for reduced birefringence of polymers stretched in rubbery (or melt) and glassy states

\begin{tabular}{cccccc}
\hline & \multicolumn{2}{c}{ Rubbery state } & & \multicolumn{2}{c}{ Glassy state } \\
\cline { 2 - 3 } \cline { 5 - 6 } & $a / 10^{3} \mathrm{~nm}^{2}$ & $b / 10^{9} \mathrm{~nm}^{4}$ & & $a / 10^{3} \mathrm{~nm}^{2}$ & $b / 10^{9} \mathrm{~nm}^{4}$ \\
\hline aPS & 29.3 & 2.04 & & 15.0 & 0.993 \\
sPS & $30.4^{\mathrm{a}}$ & $1.92^{\mathrm{a}}$ & & - & - \\
PC & 31.0 & 2.70 & & 17.3 & 3.32 \\
PET & $27.5^{\mathrm{b}}$ & $3.83^{\mathrm{b}}$ & & 25.5 & 3.25 \\
& $26.2^{\mathrm{c}}$ & $4.69^{\mathrm{c}}$ & & - & - \\
PEN & -6.83 & 9.20 & & - & -
\end{tabular}

${ }^{\text {a }} 0-48 \%$ strain-induced crystallization. $\quad$ b $2.6 \%$ strain-induced crystallization. ${ }^{c} 40.3 \%$ strain-induced crystallization.

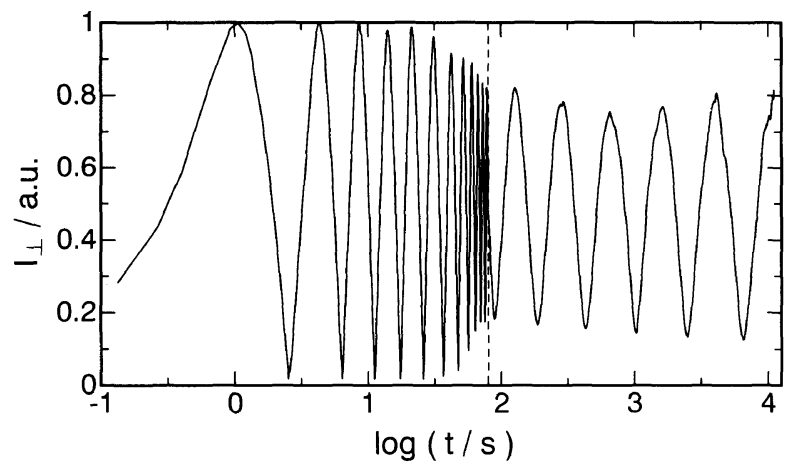

Figure 2. Typical variation of $I_{\perp}$ at $\lambda=633 \mathrm{~nm}$ in elongation and stress relaxation of PET leading to strain-induced crystallization Elongation at $85^{\circ} \mathrm{C}$ at a constant cross-head speed corresponding to the initial elongation rate $0.025 \mathrm{~s}^{-1}$. Elongation was stopped at $80 \mathrm{~s}$ (elongation ratio $=3$; indicated with dotted line) and the length was held constant.

birefringence for samples quenched in the process of stress relaxation. The temperature was lowered to the room temperature at $10^{4} \mathrm{~s}$. In Figure 2 , maximum $I_{\perp}$ at a short time was approximately equal to $I_{0}$, the $I_{\|}$on removal of the specimen from the optical system. Thus the ratio $I_{\perp} / I_{0}$ oscillated approximately between zero and unity at the early stage of elongation $(<20 \mathrm{~s})$ and then amplitude decreased. DSC measurement for quenched samples at various stages of elongation in Figure 2 revealed that the rate of crystallization suddenly increased where the amplitude of $I_{\perp}$ began to decrease. ${ }^{10}$ The crystallinity of this particular specimen was $31 \%$.

Figure 3 gives the $I_{\perp} /\left(I_{\perp}+I_{\| /}\right)$at various $\lambda$ for the specimen prepared above. The sum $I_{\perp}+I_{\| /}$closely agreed with $I_{0}$. The reduced intensity, $I_{\perp} /\left(I_{\perp}+I_{\|}\right)$, oscillated regularly but not between zero and unity. Thus eq 1 cannot be used to evaluate the birefringence. One may assume that the main origin of the oscillation is the term proportional to the right hand side of eq 1 . The birefringence values corresponding to the maxima and minima of the ratio can be obtained by $\pi d \Delta n=34800 \mathrm{~nm}$ at $\lambda=632.8 \mathrm{~nm}$, obtained by counting the maxima in Figure 2. The results for $\Delta n$ are shown in the lower panel. The function $k(\lambda)$ from these data was close to that for Figure 1 as shown later in Figure 5.

\section{Reduced Birefringence for Various Polymers}

Reduced birefringence, $k(\lambda)$, for polymers elongated in the melt or rubbery state with no crystallization is shown
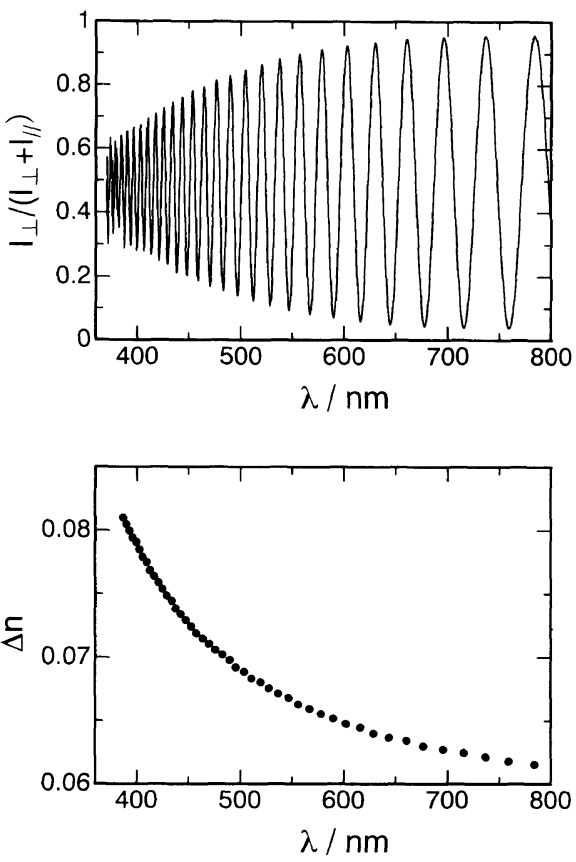

Figure 3. The ratio $I_{\perp} /\left(I_{\perp}+I_{/ /}\right)$and birefringence, $\Delta n$, at various wavelengths, $\lambda$, for the specimen shown in Figure 2 .

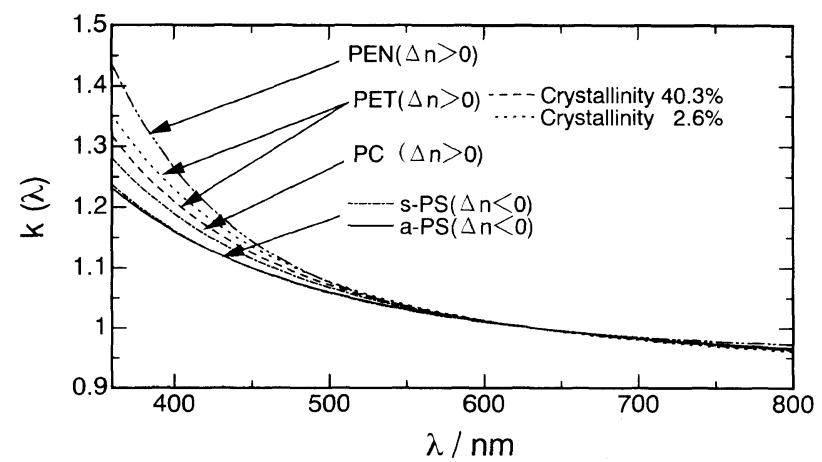

Figure 4. Reduced birefringence, $k(\lambda)$, for polymers elongated in the melt (or rubbery) state. The results for sPS are for films of various degrees of crystallization.

in Figure 4. Fitting parameters $a$ and $b$ are listed in Table II. Obviously $k(\lambda)$ is a decreasing function of $\lambda$. The results for atactic and syndiotactic polystyrene were the same and agreed with reported values. ${ }^{3}$

For PET and sPS, some specimens were partially crystallized in elongation and stress relaxation. The highest degree of crystallization as estimated with DSC measurement was $40 \%$ for PET and $48 \%$ for sPS. The function $k(\lambda)$ did not vary with the degree of crystallization for sPS. $k(\lambda)$ for PET of various degrees of crystallization is shown in Figure 5. Variation with wavelength increased slightly with crystallization. However, the difference was very small.

The results for polymers elongated in the glassy state are given in Figure 6 and Table II. $k(\lambda)$ is again a decreasing function of $\lambda . k(\lambda)$ for PET and PC is fairly close to that obtained for the melt state. The effect of $\lambda$ for PS in the glassy state is much smaller than in the melt state. 


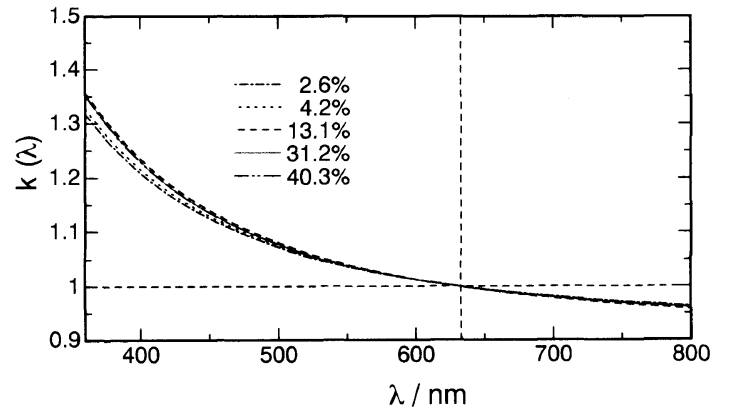

Figure 5. Reduced birefringence, $k(\lambda)$, for PET of various degrees of crystallization.

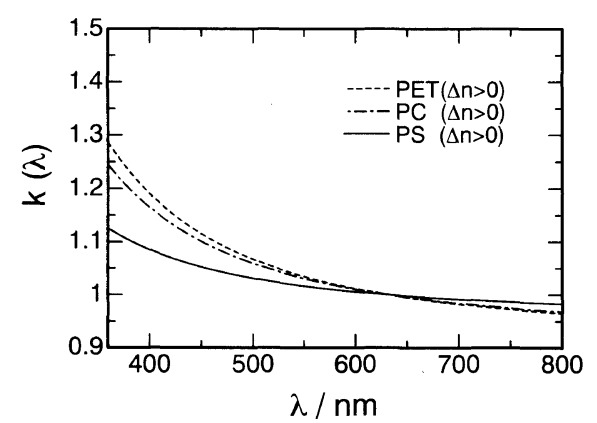

Figure 6. Reduced birefringence, $k(\lambda)$, for polymers elongated in the glassy state.

\section{DISCUSSION}

\section{On Measurements of Large Birefringence}

When birefringence is very strong, it is not possible to evaluate birefringence from the spectrum; one needs another method to determine the retardation at some $\lambda$. For the data of Figure 3, we know that the maximum just below $632.8 \mathrm{~nm}$ is the 18 th counted from the end of long $\lambda$. Without this, one could assign another choice, 19 th for example, and get a seemingly reasonable $\Delta n$ over the $\lambda$ range. Error of $\Delta n$ is about $5 \%$ at $633 \mathrm{~nm}$ and $25 \%$ at $800 \mathrm{~nm}$ in this case. Correct results for $\Delta n$ are obtained by fitting the curve (after normalizing so that it oscillates between zero and unity) by eq 1 and 2 with the parameters given in Table II for $k(\lambda)$; correct $\Delta n_{0}$ is obtained by fitting without any additional assumption. Thus the data of $k(\lambda)$ are indispensable for birefringence measurement based on white light and a spectrometer. It may be simple and rapid for measurement of strong birefringence once the $k(\lambda)$ is given for the material. ${ }^{5}$ Liquid crystalline systems are those difficult ones exhibiting strong birefringence. $\lambda$ effects seem to be carefully taken into account in some cases ${ }^{5}$ but not so much in others. ${ }^{13}$

\section{Effect of Crystallization}

Non-ideal oscillation of the ratio $I_{\perp} /\left(\mathrm{I}_{\perp}+I_{\|}\right)$as seen in Figure 3 is evidently due to strain-induced crystallization. Similar behavior was not observed for aPS even when birefringence was very high. In this regard, we would note the observation of Figure 2 in the process of elongation. When $I_{\perp}$ is continuously measured, the ratio $I_{\perp} / I_{0}$ oscillates approximately between zero and unity at the beginning and then begins to oscillate between some intermediate values. This point corresponds to the point where the stress-optical rule begins

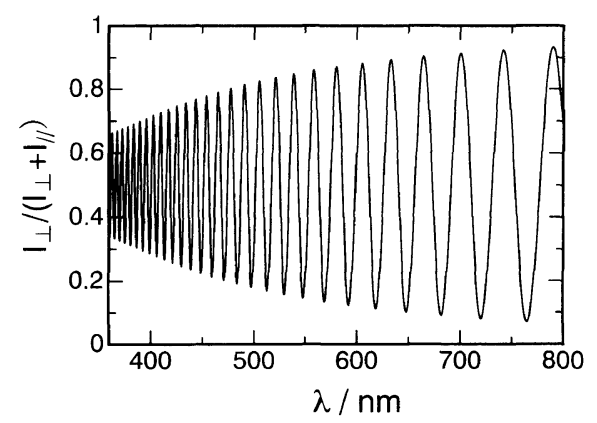

Figure 7. Results of a simple calculation of $I_{\perp} /\left(I_{\perp}+I_{\| /}\right)$for the specimen of Figure 3. Birefringence was assumed distributed with Gaussian distribution about a mean value $\Delta n_{0}(\lambda)$ and standard deviation of $\Delta n / \Delta n_{0}(\lambda)$ was assumed to be 0.0064 .

to fail and where the rate of crystallization increases. The non-ideal oscillation in Figure 3 was observed only for those films stretched beyond the above-mentioned point.

Non-ideal oscillation is not caused by the scattering of light by the sample. The sum $I_{\perp}+I_{\| /}$was very close to $I_{0}$, the transmitted intensity $I_{/ /}$on removal of the sample in all cases. Qualitative features of the curve for $I_{\perp} /\left(I_{\perp}+I_{\| /}\right)$may be mimicked by simply assuming that the material is not homogeneous so that regions of various degrees of retardation exist in the area of the light path. The cancellation of the contributions from various regions makes the maxima and minima diffuse. The effect is larger for lower $\lambda$ since the retardation is proportional to $\lambda^{-1}$, apart from the small variation of $\Delta n$ with $\lambda$, and variation of retardation is more marked at lower $\lambda$. Under this assumption, the oscillation of $I_{\perp} /\left(I_{\perp}+I_{\|}\right)$should be symmetric about the value 0.5 and the amplitude should decrease with decreasing $\lambda$.

Simple calculation is shown in Figure 7 for the specimen of Figures 3 . Here the quantity $I_{\perp} /\left(I_{\perp}+I_{\| l}\right)$ given by eq 1 was averaged under the assumption that birefringence, $\Delta n$, varied with a Gaussian distribution about a mean value $\Delta n_{0}(\lambda)$ at each $\lambda$ and the standard deviation of $\Delta n / \Delta n_{0}(\lambda)$ was 0.0064 . Evidently this well reproduces the feature of Figure 3. Similar calculations revealed that the feature might be originated by the distribution of retardation. It is more marked for films with higher birefringence with even lower crystallinity. It was not observed for amorphous films. With regard to the distribution of retardation, it may be noted in Figure 2 that the amplitude of oscillation of $I_{\perp} / I_{0}$ did not vary much in stress relaxation while crystallization increased. Thus distribution is not always determined by the degree of crystallization even with film of the same thickness. The distribution of retardation may be related to the morphology of the strain-induced crystals.

The phenomenon may be more complex, accompanied by the distribution of the orientation axis for example. In this case the curve for $I_{\perp} /\left(I_{\perp}+I_{\|}\right)$is affected more at maxima than at minima so that the central line of oscillation becomes lower than 0.5 . The distribution of orientation axis at constant retardation does not originate a $\lambda$-dependent variation of amplitude as seen in Figure 3. Our results are approximately consistent with the distribution only of retardation.

We did not detect much difference in $k(\lambda)$ for specimens accompanied by strain-induced crystallization for sPS and PET. $k(\lambda)$ for crystalline part may be close to that 

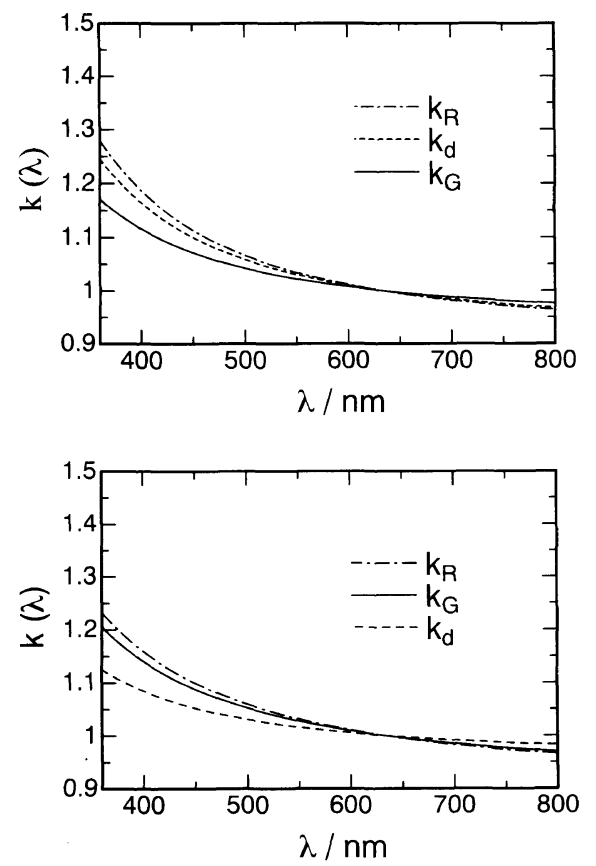

Figure 8. Comparison of $k_{\mathrm{R}}, k_{\mathrm{d}}$, and $k_{\mathrm{G}}$ for $\mathrm{PC}$ (upper panel) and aPS (lower panel).

for oriented amorphous part if not the same. This may be expected for PET for which the intrinsic birefringence $\Delta n_{0}$ is about the same for crystalline and amorphous samples. ${ }^{12}$ In such a case birefringence of crystals may be dominated by the chain orientation as for oriented amorphous polymers. The same may not be true for polymers for which $\Delta n_{0}$ varies much on crystallization.

\section{Comparison of Birefringence in Melt and Glassy States}

Birefringence in the glassy state includes contribution from the chain orientation and the rotational orientation of monomer units around the oriented axis. ${ }^{6}$ The photoelastic coefficient, $C_{\mathrm{d}}$, can be written as,

$$
C_{\mathrm{d}}=C_{\mathrm{G}}+C_{\mathrm{R}} \frac{E_{\mathrm{R}}}{E_{\mathrm{G}}}
$$

Here $C_{\mathrm{R}}$ is the stress-optical coefficient determined in the melt state and $C_{\mathrm{G}}$ is that obtained from the comparison of dynamic loss modulus and birefriengence in the glassy zone. $E_{\mathrm{R}}$ and $E_{\mathrm{G}}$ are instantaneous Young's moduli attributable to the chain orientation and monomer rotation, respectively. $C_{\mathrm{d}}$ is a combined quantity while $C_{\mathrm{R}}$ and $C_{\mathrm{G}}$ are more basic quantities related to simple orientation modes of the structural unit. ${ }^{8,9} k(\lambda)$ in Figure 4 is for the $C_{\mathrm{R}}$ and that in Figure 6 for $C_{\mathrm{d}}$.

$k(\lambda)$ for $C_{\mathrm{G}}$ can be derived from eq 4 .

$$
k_{\mathrm{G}}(\lambda)=k_{\mathrm{d}}(\lambda) \frac{C_{\mathrm{d}}}{C_{\mathrm{G}}}-k_{\mathrm{R}}(\lambda) \frac{C_{\mathrm{R}}}{C_{\mathrm{G}}} \frac{E_{\mathrm{R}}}{E_{\mathrm{G}}}
$$

$C_{\mathrm{R}}, C_{\mathrm{G}}$, and $C_{\mathrm{d}}$ at $632.8 \mathrm{~nm}$ in eq 5 are given in Table I. The ratio $E_{\mathrm{R}} / E_{\mathrm{G}}$ is 0.017 for PC and 0.0048 for PS. ${ }^{14}$ The $k_{\mathrm{G}}(\lambda)$ derived from these values and present data given in Table II is shown in Figure 8. The dependence on $\lambda$ is weaker than that of the $k_{\mathbf{R}}(\lambda)$ for these polymers. However, the difference is very small for PS. This could be fortuitous. Or it may be related to the origin of birefringence for this particular polymer.
$C_{\mathrm{R}}$ and $C_{\mathrm{G}}$ are related to the anisotropy of the polarizability tensor of the repeating unit.

$$
C_{\mathrm{R}} \propto \alpha_{1}-\frac{\alpha_{2}+\alpha_{3}}{2}, \quad C_{\mathrm{G}} \propto \frac{\alpha_{2}-\alpha_{3}}{4}
$$

Here $\alpha_{i}(i=1,2,3)$ represents the principal value of the polarizability tensor of repeating units. We assume that $\alpha_{1}$ is the value in the direction of chain axis; $\alpha_{2}$ is the larger of the two in the plane perpendicular to the chain axis. In the case of polystyrene the large negative $C_{\mathrm{R}}$ and positive $C_{\mathrm{G}}$ indicate a dominant contribution of $\alpha_{2} .{ }^{9}$ Of course the right hand sides of the proportionalities of 6 are very small portions of each component of polarizability tensor, $\alpha_{i}$, and a too simplified interpretation should be avoided. However, very similar behavior of $k_{\mathrm{R}}(\lambda)$ and $k_{\mathrm{G}}(\lambda)$ for PS may possibly represent the behavior of $\alpha_{2}$ for this polymer.

\section{CONCLUSION}

The effect of wavelength of light, $\lambda$, on the straininduced birefringence, $\Delta n$, was measured for uniaxially elongated films of polymers. The results for various polymers were represented by the reduced birefringence, $k(\lambda) \equiv \Delta n(\lambda) / \Delta n(632.8 \mathrm{~nm})$, which fitted well the quadratic function of $\lambda^{-2}$. The data of $k(\lambda)$ can be utilized in birefringence measurement with white light and a spectrometer.

$k(\lambda)$ was always a decreasing function of $\lambda$ for the polymers studied. For PC and PET $\lambda$-dependence was much stronger when strain was applied in the rubbery state (corresponding to SOC) than in the glassy state (corresponding to PEC). The difference was very small for PS. These results may be explained with a model theory in which the SOC is associated solely with the chain orientation while the PEC is affected also by the monomer rotation around the chain axis induced by deformation of the material in glassy state.

Strain-induced crystallization led to complicated behavior of transmitted light intensity at short $\lambda$ when the material was inserted between a pair of parallel or crossed polarizers. Anomalous behavior could be accounted for by assuming a slight distribution of retardation around the mean value at each $\lambda$. The straininduced crystallization in sPS and PET did not significantly affect $k(\lambda)$. This probably indicates that the origin of the birefringence of strain-induced crystals is mainly the chain orientation for the polymers exhibiting strong birefringence in amorphous state.

Acknowledgment. This study was supported by a Grant-in-Aid for Scientific Research (No. 09450361) from the Ministry of Education, Science, Sports and Culture of Japan.

\section{REFERENCES}

1. H. Janeschitz-Kriegl, "Polymer Melt Rheology and Flow Birefringence," Springer-Verlag, Berlin, 1983.

2. G. G. Fuller, "Optical Rheometry of Complex Fluids," Vol. 22, Oxford University Press, New York, N.Y., 1995.

3. E. F. Gurnee, J. Polym. Sci., A-2, 5, 817 (1967).

4. J. I. Kroschwitz, Ed., "Concise Encyclopedia of Polymer Science and Engineering," Wiley, New York, N.Y., 1990, p 684. 
5. K. Hongladarom, W. R. Burghardt, S. G. Baek, S. Cementwala, and J. J. Magda, Macromolecules, 26, 772 (1993).

6. T. Inoue, H. Okamoto, and K. Osaki, Macromolecules, 24, 5670 (1991)

7. M. Doi and S. F. Edwards, "The Theory of Polymer Dynamics," Clarendon, Oxford, 1986.

8. K. Osaki, H. Okamoto, T. Inoue, and E.-J. Hwang, Macromolecules, 28, 3625 (1995).

9. T. Inoue, H. Matsui, and K. Osaki, Rheol. Acta, 36, 239 (1997).
10. T. Inoue, H. Matsui, S. Murakami, S. Kojiya, and K. Osaki, Polymer, 38, 1215 (1997).

11. T. Inoue, T. S Ryu, K. Osaki, and T. Takebe, J. Polym. Sci., Polym. Phys. Ed., in press.

12. D. S. Ryu, T. Inoue, and K. Osaki, Polymer, 39, 2515 (1998)

13. F. Beekmans and A. Porthuma de Boer, Macromolecules, 29, 8726 (1996).

14. T. Inoue, E. J. Hwang, and K. Osaki, J. Rheol., 36, 1737 (1992). 